\title{
Ground States for Mass Critical Two Coupled Semi-Relativistic Hartree Equations with Attractive Interactions
}

\author{
Thi-Anh-Thu DOAN ${ }^{1}$ \\ ${ }^{1}$ Ton Duc Thang University Faculty of Mathematics and Statistics
}

December 15, 2021

\begin{abstract}
We prove the existence and nonexistence of $\$ \mathrm{~L}^{\wedge}\{2\}\left(\backslash\right.$ mathbb $\left.\mathrm{R}^{\wedge} 3\right) \$$-normalized solutions of two coupled semi-relativistic Hartree equations, which arisen from the studies of boson stars and multi-component Bose-Einstein condensates. Under certain condition on the strength of intra-specie and inter-specie interactions, by proving some delicate energy estimates, we give a precise description on the concentration behavior of ground state solutions of the system. Furthermore, an optimal blowing up rate for the ground state solutions of the system is also proved.
\end{abstract}

\section{Hosted file}

blow-up-mixture-boson-stars.pdf available at https://authorea.com/users/451212/articles/ 549436-ground-states-for-mass-critical-two-coupled-semi-relativistic-hartree-equationswith-attractive-interactions

\section{Hosted file}

blow-up-mixture-boson-stars.tex available at https://authorea.com/users/451212/articles/ 549436-ground-states-for-mass-critical-two-coupled-semi-relativistic-hartree-equationswith-attractive-interactions 